044 DRAMATIC DELAYED RECOVERY FOLLOWING SEVERE ANTI-NMDAR ENCEPHALITIS

${ }^{1}$ Allycia MacDonald*, ${ }^{2}$ Nerissa Jordan, 'Wai Leong. 'Department of Neurology, Royal Perth Hospital, Perth, WA, Australia; ' ${ }^{2}$ epeartment of Neurology, Fiona Stanley Hospital, Murdoch, WA, Australia

10.1136/jnnp-2019-anzan.39

Introduction Long term outcomes in anti-N-methyl-D-aspartate receptor (NMDAR) encephalitis are reported to be favourable in $80 \%$ of cases, but there is little information regarding recovery following prolonged severe neurological disability.

Case 1 A 16-year-old female presented with headache, fever and acute encephalopathy. Anti-NMDAR antibodies were positive, with an ovarian teratoma. Treatment included oophorectomy, plasmapheresis, corticosteroids, cyclophosphamide, intravenous and intrathecal rituximab, and alemtuzumab. After 15 months hospitalisation, she remained in a vegetative state, with tracheostomy and percutaneous gastrostomy tubes, on multiple anticonvulsants. She received no further immunotherapy. MRI brain 2 years after illness onset demonstrated severe generalised cerebral atrophy. Three years after onset, she began to improve. At $5+$ years she was seizure-free without anticonvulsants, fully independent and ambulatory, without overt cognitive deficits or personality change. MRI brain at $51 / 2$ years demonstrated recovery of cerebral volume to within normal limits, but residual hippocampal atrophy.

Case 2 A 21-year-old female presented with headache, fever and personality change. NMDAR-antibodies were positive. A malignant ovarian teratoma was treated with salpingoophorectomy and chemotherapy (bleomycin/etoposide/cyclophosphamide). She received plasmapheresis, immunoglobulins, corticosteroids, intravenous and intrathecal rituximab, and bortezomib. She was finally discharged after 21 months hospitalisation with tracheostomy and gastrostomy tubes, severe behavioural disturbance, minimal functional limb movement, and fully dependent care needs. Clinical recovery began $21 /$ 2 years after disease onset, and at 4 years she is fully independent with residual cognitive deficits, engaging in volunteer work.

Conclusions These cases highlight the potential for dramatic delayed clinical improvement despite prolonged severe neurological disability in anti-NMDAR encephalitis.

\section{PRIMARY CUTIBACTERIUM ACNES CENTRAL NERVOUS SYSTEM INFECTION CAUSING FOCAL SEIZURES: AN UNUSUAL PRESENTATION OF A RARE DISEASE}

${ }^{1}$ Sophie E Waller*, ${ }^{2}$ Sarah Browning, ${ }^{1}$ Elizabeth Pepper. ${ }^{1}$ Department of Neurology, John Hunter Hospital, New Lambton Heights, NSW, Australia; ${ }^{2}$ Department of Infectious Diseases, John Hunter Hospital, New Lambton Heights, NSW, Australia

\subsection{6/jnnp-2019-anzan.40}

Introduction Cutibacterium acnes is a Gram positive, anaerobic bacterium of low pathogenic potential that forms part of the normal cutaneous flora. Although most often identified as a contaminant in culture of microbiological specimens, it is commonly implicated in both postoperative wound and implantable device infection. Neurosurgical device infections secondary to $C$. acnes are well recognised and are likely secondary to bacterial contamination from the skin during surgery. Indolent infection characterised by delayed presentation of weeks to months following intervention is common. C. acnes infection involving the central nervous system (CNS) in the absence of previous neurosurgical intervention is rare, but has been described following dental or mastoid infections and following facial trauma. A further case series has reported de novo C. acnes CNS infection occurring in the absence of these recognised risk factors, but with clinical features of meningitis being common to all.

Methods and results We describe a unique case of primary C. acnes extra-dural collection in a previously well patient with no neurosurgical history presenting with sub-acute focal seizures and progressive focal leptomeningeal thickening on MRI.

Conclusion C. acnes CNS infection can occur in the immunocompetent and in the absence of neurosurgical intervention.

\section{A CRITICAL REVIEW OF BIOMARKERS FOR HEREDITARY SPASTIC PARAPLEGIA}

${ }^{1,2,3}$ Sue-Faye Siow ${ }^{*}{ }^{1,2}$ Gautam Wali, ${ }^{4,1,2}$ Carolyn Sue, ${ }^{4,1,2,5}$ Kishore R Kumar. ${ }^{1}$ Northern Clinical School, University of Sydney, Sydney, NSW, Australia; ${ }^{2}$ Neurogenetics, Kolling Institute, Sydney, NSW, Australia; ${ }^{3}$ Clinical Genetics, Royal North Shore Hospital, Sydney, NSW, Australia; ${ }^{4}$ Neurology, Royal North Shore Hospital, Artarmon, NSW, Australia; ${ }^{5}$ Garvan Institute of Medical Research, Sydney, NSW, Australia

\subsection{6/jnnp-2019-anzan.41}

Introduction Hereditary spastic paraplegia (HSP) is a rare neurodegenerative condition characterised by lower limb weakness and spasticity. Currently, treatment is symptomatic and there is no disease modifying therapy. Though candidate therapeutic agents have been identified, sensitive biomarkers to measure treatment efficacy in clinical drug trials are lacking. There are many challenges in the search for appropriate biomarkers including the rarity of HSP, clinical and genetic heterogeneity of HSP and slow disease progression.

Methods We performed a search on PubMed and Medline using the search terms ('hereditary spastic paraplegia' OR 'spastic paraparesis') AND 'biomarker"'. We searched the reference lists of relevant articles to identify further studies. We collected data on number of participants, HSP genotype, methodology, and outcomes.

Results 72 papers were identified: 2 on Rating scales, 9 on gait analysis, 33 on neurophysiological measures, 23 on neuroimaging markers and 5 on biochemical markers. The studies reviewed demonstrated variation in methodologies and outcomes, including mixed genotype (41/72 papers) and genotypespecific (31/72 papers) patient cohorts, varied neurophysiological techniques and different outcome measures. 68/72 studies reviewed had small patient numbers ( $<50$ patients). All potential biomarkers reviewed were able to differentiate HSP patients from controls. Only diffusion tensor imaging (DTI) parameters showed significant correlation with disease severity. 
Conclusion Although useful as diagnostic biomarkers, further studies are required to evaluate these potential biomarkers longitudinally and to assess their reliability as surrogates for underlying neurodegenerative changes and clinical disease severity. DTI showed the most promise as a biomarker for disease severity in HSP.

\section{ACUTE LUMBOSACRAL PLEXOPATHY AFTER BLOOD LOSS IN A PATIENT WITH BILATERAL COMMON ILIAC ARTERY OCCLUSION}

Matthew Katz*, Pamela McCombe. Royal Brisbane and Women's Hospital, Brisbane, QLD, Australia

10.1136/jnnp-2019-anzan.42

Introduction We present a case of a 56 year old male with bilateral, asymmetric leg weakness from ischemic lumbosacral plexopathy. This followed acute blood loss from an episode of self-harm, which occurred in the setting of chronic thrombotic occlusion of bilateral common iliac artery (CIA) stents.

Methods A detailed history, examination and neurophysiology study were performed in addition to reviewing case notes and results of other investigations. A brief literature review was also completed.

Results The patient presented with acute blood loss following self-inflicted lacerations to his anterior neck and both wrists. He had asymmetric flaccid, areflexic weakness of his legs with decreased sensation over the right foot and absent distal leg pulses. Magnetic resonance imaging (MRI) of brain and whole spine was normal. Computed tomography angiogram (CTA) revealed significant distal aortic atherosclerotic disease with complete occlusion of bilateral CIA stents. The patient underwent endovascular re-stenting of both common iliac arteries with a good angiographic result. An electromyogram performed one month later was supportive of a lumbosacral plex-opathy, mainly on the right.

Only three cases of acute ischemic lumbosacral plexopathy have been reported in the setting of aorto-iliac occlusive disease. $^{1-3}$

Conclusion Ischemic injury to the lumbosacral plexus is rare, especially outside the surgical setting as seen in this case. It is important to consider this cause in any patient presenting with rapid onset asymmetric, bilateral leg weakness as early recognition and reperfusion may prevent further damage and reduce long-term disability.

\section{REFERENCES}

1. Schreuder AH, Fennis TF, Teijink JA, Koehler PJ. Lumbosacral plexopathy associated with aortoiliac occlusive disease. J Neurol. 2007;254(6):803-5.

2. Chhetri SK, Lekwuwa G, Seriki D, Majeed T. Acute flaccid paraparesis secondary to bilateral ischaemic lumbosacral plexopathy. QJM. 2013;106(5):463-5.

3. Yun S. Ischaemic lumbosacral plexopathy following an acute thrombosed abdominal aortic aneurysm mimicking stroke: A case report. Hong Kong Journal of Emergency Medicine. 2016;23(2):52-6.

\section{HARDING'S DISEASE: AN IMPORTANT MS MIMIC}

${ }^{1}$ Stuti Joshi*, ${ }^{2}$ Allan Kermode. ${ }^{1}$ Sir Charles Gairdner Hospital, Stirling, WA, Australia; ${ }^{2}$ Perron Institute, Sir Charles Gairdner Hospital, Nedlands, WA, Australia

10.1136/jnnp-2019-anzan.43

Introduction Leber's hereditary optic neuropathy is a mitochondrially-inherited disorder characterized by bilateral, painless visual loss, which leads to severe optic atrophy. ${ }^{1}$ LHON can be associated with an MS-like illness referred to as Harding's disease. ${ }^{2}$

We report two siblings, who both harbour the 11778 mtDNA mutation, but manifest markedly different clinical phenotypes; a male with classical LHON and a female with Harding's disease.

Methods and Results A 61-year-old female, who was diagnosed with MS 22 years ago was referred to our service for a second opinion. She developed unilateral painless visual loss in her 20's, was diagnosed with optic neuritis and treated with corticosteroids with some recovery. A second episode of more severe visual loss at age 39 left her with visual impairment to less than finger counting. 4 years later, she had an episode of dysarthria and gait ataxia. MRI showed multifocal white matter lesions involving the juxta-cortical and periventricular regions, cerebellar peduncle and cervical cord. Targeted views of the optic pathways showed hyperintensity of the left optic nerve, with involvement extending into the optic canal.

The patient has one brother who was diagnosed with LHON at age 37 after presenting with severe painless bilateral sequential visual loss. Genetic testing of the index patient confirmed the presence of the same mutation identified in her brother.

Conclusion LHON and Harding's disease demonstrate a great degree of variability in clinical phenotype and penetrance between males and females as well as individuals within the same family. ${ }^{3}$ While there is no evidence for screening MS cohorts for the LHON, consider genetic testing in patients with severe and persistent bilateral visual loss or with a suggestive family history. 4

\section{REFERENCES}

1. Harding $A E$, Sweeney MG, Miller DH, Mumford CJ, Kellar-Wood H, Menard D, McDonald WI, Compston DA. Occurrence of a multiple sclerosis-like illness in women who have a Leber's hereditary optic neuropathy mitochondrial DNA mutation. Brain. 1992 August;115 ( Pt 4):979-89.

2. Palace J. Multiple sclerosis associated with Leber's Hereditary Optic Neuropathy. J Neurol Sci. 2009 November 15;286(1-2):24-7. Review.

3. Pfeffer G, Burke A, Yu-Wai-Man P, Compston DAS, Chinnery PF. Clinical features of MS associated with Leber hereditary optic neuropathy mtDNA mutations. Neurology. 2013;81(24):2073-2081.

4. Yu-Wai-Man P, Chinnery PF. Leber hereditary optic neuropathy. In: Pagon RA MP, Adam Ardinger HH eds. GeneReviews. Seattle, WA: University of Washington, Seattle; 2013. Accessed May 7, 2018.

\section{REAL WORLD EVIDENCE (RWE) ON IMPACT OF AGE ON LONG-TERM PERSISTENCE TO DISEASE MODIFYING THERAPIES (DMTS) IN RELAPSING-REMITTING MULTIPLE SCLEROSIS (RRMS) IN AUSTRALIA}

${ }^{1}$ Andrew Kornberg* ${ }^{2}$ Tim Spelman, ${ }^{3}$ Mark Schulz, ${ }^{3}$ Birendra Arora, ${ }^{4}$ Eric Chung ${ }^{5}$ Prabjot Juneja, ${ }^{3}$ Robert Walker, ${ }^{6}$ Anneke van der Walt, ${ }^{6}$ Helmut Butzkueven. ${ }^{1}$ Royal Children's Hospital, Parkville, VIC, Australia; ${ }^{2}$ Department of Medicine, University of Melbourne, Melbourne, VIC, Australia; ${ }^{3}$ Novartis, North Ryde, NSW, Australia; ${ }^{4}$ Prospection Pty Ltd, Australian Technology Park, Eveleigh, NSW, Australia; ${ }^{5}$ Prospection Pty Ltd, Australian Technology Park, Eveleigh, NSW, Australia; ${ }^{6}$ MS and Neuroimmunology Group, Central Clinical School, Monash University and Alfred Hospital, Melbourne, VIC, Australia

\subsection{6/jnnp-2019-anzan.44}

Background Age has been independently associated with higher rates of treatment discontinuation in multiple sclerosis.

Objective The current study examines the impact of age on persistence for all reimbursed DMTs for RRMS in Australia. Methods The Pharmaceutical Benefits Scheme (PBS) 10\% sample supplied by the Department of Human Services was used 